\title{
A practical guide to the use of consumer-level digital still cameras for precise stereogrammetric in situ assessments in aquatic environments
}

\author{
Matthias Wehkamp and Philipp Fischer* \\ Alfred-Wegener-Institut, Helmholtz Centre for Polar and Marine Research, Biologische Anstalt Helgoland, \\ Kurpromenade 207, 27498 Helgoland, Germany
}

Received 12 August 2013; Accepted 18 June 2014

\section{Abstract}

Scientists planning to use underwater stereoscopic image technologies are often faced with numerous problems during the methodological implementations: commercial equipment is too expensive; the setup or calibration is too complex; or the imaging processing (i.e. measuring objects in the stereo-images) is too complicated to be performed without a time-consuming phase of training and evaluation. The present paper addresses some of these problems and describes a workflow for stereoscopic measurements for marine biologists. It also provides instructions on how to assemble an underwater stereo-photographic system with two digital consumer cameras and gives step-by-step guidelines for setting up the hardware. The second part details a software procedure to correct stereo-image pairs for lens distortions, which is especially important when using cameras with non-calibrated optical units. The final part presents a guide to the process of measuring the lengths (or distances) of objects in stereoscopic image pairs. To reveal the applicability and the restrictions of the described systems and to test the effects of different types of camera (a compact camera and an SLR type), experiments were performed to determine the precision and accuracy of two generic stereo-imaging units: a diver-operated system based on two Olympus Mju 1030SW compact cameras and a cable-connected observatory system based on two Canon 1100D SLR cameras. In the simplest setup without any correction for lens distortion, the low-budget Olympus Mju $1030 S W$ system achieved mean accuracy errors (percentage deviation of a measurement from the object's real size) between 10.2 and $-7.6 \%$ (overall mean value: $-0.6 \%$ ), depending on the size, orientation and distance of the measured object from the camera. With the single lens reflex (SLR) system, very similar values between $10.1 \%$ and $-3.4 \%$ (overall mean value: $-1.2 \%$ ) were observed. Correction of the lens distortion significantly improved the mean accuracy errors of either system. Even more, system precision (spread of the accuracy) improved significantly in both systems. Neither the use of a wide-angle converter nor multiple reassembly

* Contact author. E-mail address: philipp.fischer@awi.de of the system had a significant negative effect on the results. The study shows that underwater stereophotography, independent of the system, has a high potential for robust and non-destructive in situ sampling and can be used without prior specialist training.

Keywords: cabled observatories, COSYNA, stereogrammetry, in situ length measurements, scientific diving

\section{Introduction}

Stereophotography has been continuously applied as a tool in the aquatic sciences since the early $1960 \mathrm{~s}$ (e.g. Boyce, 1963; Christie and Green, 1982; Leatherdale and Turner, 1983; Bräger and Chong, 1999; Bythell et al., 2001; Brandou et al., 2007; Waite et al., 2007). As in terrestrial ecology, stereophotography enables reference-free and non-invasive measurements of object coordinates in the $3 \mathrm{D}$ realm, thus eliminating the need for a parallel estimation of quantitative parameters such as distance or volume (e.g. Cullen et al., 1965; Rorslett et al., 1978; Van Rooij and Videler, 1996; Bräger and Chong, 1999; Costa et al., 2006; Williams et al., 2010). Photographic assessments of time series of communities also allow the estimation of quantitative derivatives, including growth or productivity over time, using an empirically determined relationship between biomass and linear measurements (Williams, 1969; Rorslett et al., 1978; Fischer et al., 2007; Williams et al., 2010, Doya et al., 2014).

The primary applications of stereo-image techniques in marine biology are in abundance and habitat surveys (Shortis et al., 2009). A range of other marine biology applications also routinely use stereoimaging techniques (photo as well as video), including: fish monitoring (Ruff et al., 1995; Van Rooij et al., 1995; Li et al., 1996; Tillett et al., 2000; Harvey et al., 2003; Costa et al., 2006; Costa et al., 2009; 
Rooper et al., 2010; Bower et al., 2011; Torisawa et al., 2011); determination of the 3D structure of fish schools (Cullen et al., 1965; Pitcher, 1975; Dill et al., 1981; Sawada et al., 2009); determination of the influence of substrate complexity on the interaction between fish and the artificial substrate (Fischer et al., 2007); and surveys of the benthos and the sea floor (Emerey et al., 1965; Evans and Norris, 1997; Chong and Stratford, 2002; Jasiobedzki et al., 2008; Shortis et al., 2008; Svane et al., 2009).

Excellent summaries of the applications of stereophotography and videogrammetry to marine biology have been published (Williams, 1969; Rorslett et al., 1978; Fischer et al., 2007; Williams et al., 2010; Aguzzi et al., 2012), which demonstrate the usefulness of both stereogrammetry-based videos and still photography in assessing the metric traits of fish stocks, for example in 'untrawlable' habitats. Stereoscopic assessments, furthermore, provide a valuable tool for SCUBA-supported non-destructive rapid assessment surveys and monitoring programmes, and are especially applicable to the assessment of endangered species, which are protected against capture (Shortis et al., 2007).

A major restriction in the application of underwater stereophotography as a routine tool for the assessment of species metric traits, however, is the apparent complexity of setting up an adequate stereoscopic camera system and processing the images so that exact measurements of objects (e.g. the length of fish or any other distances) can be achieved. Even though an increasing number of software packages are available (i.e. based on MATLAB) to simplify the process of stereoscopic image analysis (Williams et al., 2010), the application of these products especially to larger numbers of stereoscopic image pairs - is still challenging. This is mainly because a series of different steps, each with specific technology, is necessary to finally achieve precise measurements (e.g. of an object's length) from a set of stereo-images. These steps include the assembly of a stereoscopic still camera system, the correction for lens distortions of the two cameras, calibration of the system and the final step of the measurement of objects, which are mapped onto the image pairs.

To determine an object's length on a set of stereoimages, a minimum of three values are required: (a) the distance between the lens of the camera and the chip plane (intrinsic geometric measure); (b) the distance between the optical axes of the two cameras (extrinsic geometric measures); and (c) the $x y$-coordinates of two points (e.g. the mouth and the caudal peduncle of a fish) on both images.

Unfortunately, the camera parameters $(a)$ and $(b)$ are normally not available for standard consumer cameras and, therefore, have to be determined experimentally for each specific camera system. Furthermore, many algorithms used to calculate an object's length based on a pair of stereoscopic images require the parallelism of the optical axis of the two cameras. Although this was hard to achieve previously with analogue photography, digital imaging enabling the possibility of transferring stereoscopic image pairs without dismounting them from the rack provides a comparatively easy way to preadjust the optical axes of two cameras to approximate parallelism, which is often sufficient for many stereo-optical assessments (Fischer et al., 2007).

Furthermore, optical systems today are often cheap and have a variety of lens distortions. While this is hardly a problem in normal photography, it might result in a significant loss of accuracy in quantitative object measurements with such systems. Lens distortion appears in virtually every camera lens. The type of distortion (radial or decentring) depends largely on the design of the lens, the position of the object image in the photo or (as is mostly the case for standard consumer and compact cameras) on the quality of the manufacture and the assembly of the camera.

In addition, the high turbidity (in shallow coastal waters) often requires the use of a wide-angle converter (WAC) in underwater photography. The shorter focal length and the larger angle of view of a WAC allow for a shorter distance between the camera and the object, and therefore fewer particles in the water that diffuse the light. Unfortunately, WACs also often have a much stronger lens aberration compared with standard lens systems. Therefore, the object distortion caused by lens aberration can be a major problem that significantly reduces the accuracy and therefore the statistical power (Harvey et al., 2001) of stereoscopic measurements underwater.

To compensate for lens aberrations, images can be corrected prior to taking exact measurements. During the process of removing lens distortion, all pixels of the stereo-images are shifted such that the lines and position on the images are mapped correctly with respect to their true positions relative to each other, assuming no lens distortion.

The present study had two main goals: (a) to provide a practical, reliable, and - for ecological in situ studies - sufficiently precise procedure to assess dimensions (e.g. body lengths of fish or decapod crustaceans) by stereo-photographic evaluation; and $(b)$ to allow aquatic ecologists, without specific mathematical or engineering training in stereoimage assessment or subsequent analysis, to apply this technology to ecological studies. Therefore, the present paper describes the general assemblage of two different stereo-camera systems: one cheap system comprising two Olympus consumer cameras; and 
one more sophisticated system comprising two single-lens reflex (SLR) Canon 1100D cameras. It then focuses on the procedures for calibrating the systems and finally provides data on the measurement accuracy and precision achieved with the two different camera setups.

\section{Materials and methods}

\subsection{The camera systems}

The experiments used two different camera systems to test whether the procedures for calibration, removal of lens distortions and measurement presented in the present study are independent of the type of camera and the way the cameras are assembled into a stereoscopic system. The basic principle of both systems was that two identical cameras were mounted with standard tripod screws onto a single base-plate made of PVC or metal and were then adjusted to optical axis parallelism as much as possible.

The first camera system was a low-cost system (Fig 1, Table 1) consisting of two identical Olympus Mju 1030SW still cameras in standard underwater housings from Olympus (PT-O43; waterproof to $40 \mathrm{~m})$. The two cameras were mounted on a baseplate with a horizontal distance of $139 \mathrm{~mm}$ between them, which was found to be the narrowest distance that would allow the cameras to be removed from the housings without removing the housings from the base-plate itself.

The base-plate was mounted onto a rectangular rack made of $20 \mathrm{~mm}$ Bosch-Rexroth ${ }^{\mathrm{TM}}$ aluminium sectional rail. This allowed for better handling of the system by divers, protected against accidental damage and allowed the mounting of supplemental parts, including a flashlight, permanent illumination and various pieces of equipment needed for the latter in in situ applications. The total cost

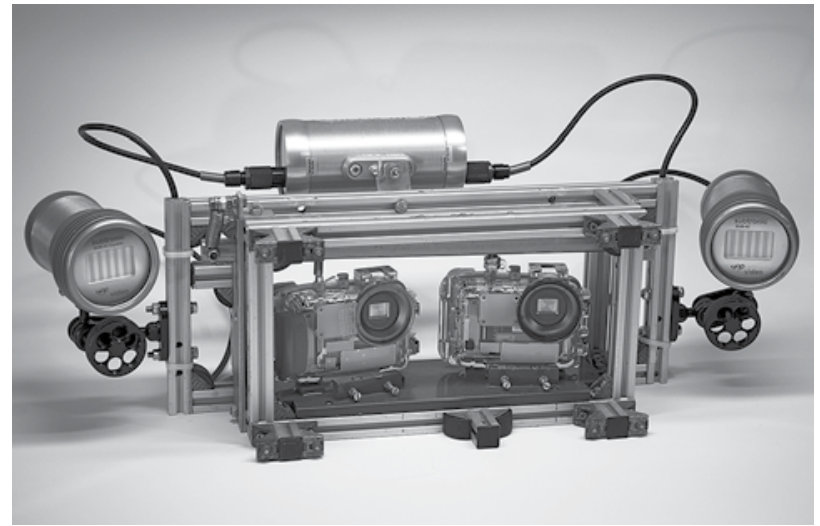

Fig 1: Portable underwater stereo-camera system, with two identical low-cost cameras (Olympus Mju 1030sw). The system can be equipped with a permanent light system (as shown) or a system flashlight

of the system, including the flashlight, was about $€ 1,800$ (without WACs) and $€ 2,300$ with WACs.

The SLR camera system (Fig 2a, Table 1) consisted of two identical Canon 1100D still cameras with standard lens types $(18-54 \mathrm{~mm})$ mounted in a single housing of stainless steel (Fig 2b), which was specifically made for long-term exposure. The two cameras were separated by a horizontal distance of $172 \mathrm{~mm}$ on the base-plate (the narrowest distance that would allow cables to be removed and installed without removing the cameras from the base-plate). Except for the focus function, which had to be set to manual (the autofocus function does not allow proper synchronisation), exposure and aperture values could be selected according to the requirements of the experiment and adjusted via remote connection.

The camera system, including triggering and image download, was controlled by a microcomputer mounted in the housing, which was connected via transmission control protocol/Internet protocol

Table 1: Main characteristics of the two still camera systems used

\begin{tabular}{|c|c|c|}
\hline & Olympus Mju 1030sw & Canon EOS 1100D \\
\hline $\begin{array}{l}\text { Manufacture } \\
\text { Image sensor } \\
\text { Sensor type } \\
\text { Maximum output resolution } \\
\text { Shutter speed } \\
\text { ISO rating } \\
\text { Optical focal length (35mm equivalent) } \\
\text { Maximum lens aperture } \\
\text { Closest focusing distance } \\
\text { Auto-focus } \\
\text { Manual focus } \\
\text { Image formats } \\
\text { Display size }\end{array}$ & $\begin{array}{l}\text { Olympus } \\
10 \mathrm{MP}, 6.2 \times 4.6 \mathrm{~mm} \\
\text { CCD } \\
3,648 \times 2,736 \\
1 / 1,000-4 \mathrm{~s} \\
80-1,600 \\
28-102 \mathrm{~mm} \\
\text { f3.5-5.1 } \\
20 \mathrm{~cm} \\
\text { iESP, spot, } \\
\text { No } \\
\text { JPEG } \\
2.7^{\prime \prime}\end{array}$ & $\begin{array}{l}\text { Canon } \\
12.2 \mathrm{MP}, 22.2 \times 14.7 \mathrm{~mm} \\
\text { CMOS } \\
4,272 \times 2,848 \\
1 / 4,000-30 \mathrm{~s} \\
100-6,400 \\
29-88 \mathrm{~mm}^{*} \\
\text { f3.5-5.6* } \\
25 \mathrm{~cm}^{*} \\
\text { Al Focus, Al Servo, One shot } \\
\text { Yes } \\
\text { JPEG, DCF-format (2.0), RAW, DPOF } \\
2.7^{\prime \prime}\end{array}$ \\
\hline${ }^{*}$ Canon lens EF-S 18-55 1:3.5 - 5 & & \\
\hline
\end{tabular}




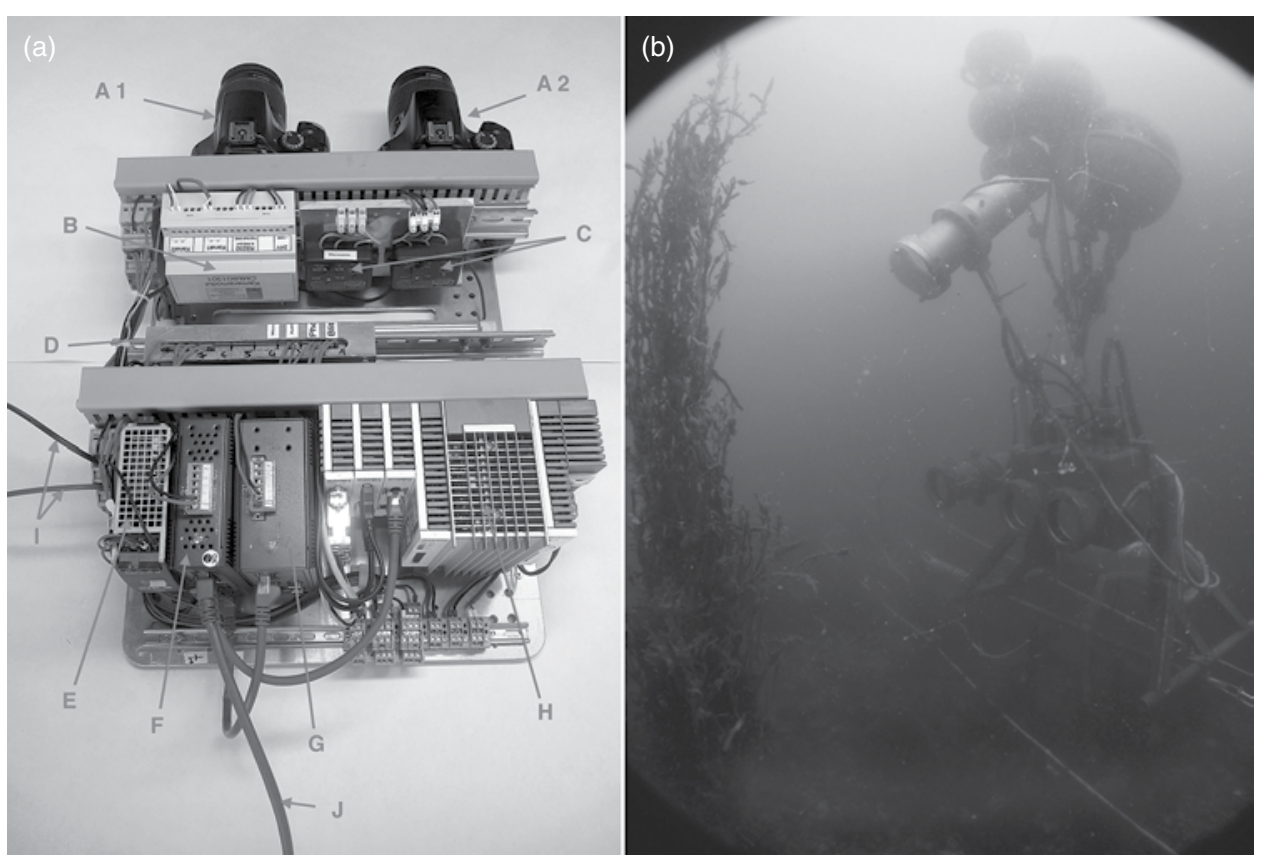

Fig 2: (a) High-end stationary underwater stereo-camera system with two identical SLR cameras (Canon 1100D) mounted on a single base plate. The individual parts are A1 and A2: SLR Canon Cameras D1100; B: electronic synchronised trigger system of the two cameras; C and E: DC/DC power supply for 24V, 9V and 6V; F: network switch; D: on/off relays board for remote power supply control of various components of the systems; G: TCP/IP-RS232 converter; H: industrial computer for system control and remote access; and (b) the system mounted in a single housing made of stainless steel, installed as a fish-measuring device at Helgoland at a water depth of $5 \mathrm{~m}$

(TCP/IP) to a land-based control centre, where the images were stored and processed.

The total cost of the SLR system, including flashlight and network connection, was about $€ 25,000$ without the land-based control station and the underwater cable connection. The latter was realised within the framework of Coastal Observing System for Northern and Arctic Seas (COSYNA; Doerffer et al., 2008), a large-scale German infrastructure project for coastal sciences managed by the Helmholtz Centre, Geesthacht, in close cooperation with the Alfred Wegener Institute, the Helmholtz Centre for Polar and Marine Research. Together with others, COSYNA hosts the subproject 'COSYNA Underwater Nodes' and has installed an underwater node in 2012 off Helgoland at a water depth of about $11 \mathrm{~m}$. The node system is connected via a cable to a landbased control station and allows for year-round online measurements of the main abiotic and biotic variables (temperature, salinity, depth (tide), turbidity, oxygen, Chl-a fluorescence, 3D current) in real time $(1 \mathrm{~Hz})$. The low-cost system tested in the present study was always diver operated, and the stationary SLR system was attached to the COSYNA underwater node system and was operated via remote control.
For both stereoscopic systems (the SLR and lowcost systems), the optical axes were adjusted to be as parallel as possible prior to the assessments, following the procedure of Klimley and Brown (1983), adapted for digital cameras (Fischer et al., 2007).

For the present study, a bearing line was drawn on the base-plate of the cameras halfway between the two optical axes and parallel to them. The entire system was then aligned such that an object at 'infinity' distance from the camera was targeted by this bearing line (Klimley and Brown, 1983).

In the next step, two images were taken by both cameras, transferred to a computer and opened with image software that provides the $x y$-coordinates of the cursor in pixels relative to the images' dimensions (IrfanView freeware available at www.irfanview.de). The horizontal deviation of the 'object in infinity' on both images was then determined in pixels. On.f the two cameras was repeatedly horizontally adjusted on the base-plate until the object at distance 'infinity' was projected into both images at the same horizontal position (Klimley and Brown, 1983). Because the optical axis of non-calibrated consumer cameras almost never coincides with the centre of the pixel array, this procedure unfortunately does not allow for a correction of the principal 
Table 2: Sequential steps in stereoscopic image processing from camera calibration to measuring objects on stereo-images

Process
left and the right camera: determining a camera's intrinsic parameters and the respective lens distortion matrix
Step 7-8: Determining the stereo system's extrinsic parameters, adapting the lens distortion matrixes to the stereo system and verifying the calibration process

Step 9-13: Rectification of larger numbers of stereo-images pairs

\section{Step number and task}

1) Take about 20 calibration images and save them in a single folder.

2) Rename the images according to the 'calib_gui' name convention with a fixed prefix (e.g. 'left_' or 'right_') and a sequential number beginning with 1. A corresponding number of images from the left camera and the right camera must be in the folder.

3) Load the left (right) images in the routine 'calib_gui'.

4) Extract grid corners.

5) 'Calibration' of the left (right) camera and 'Save' the results. In this step, a file called 'calib_result.mat' is produced in the folder where the images are located. This file has to be manually renamed in 'calib_result_left.mat' ('calib_results_right. mat').

6) Repeat steps 3 to 5 with the right (or left) images.

7) Read the left and right calibration files with 'Load left and right calibration files'.

8) Run the stereo calibration and save the results. In this step, a file called 'Calib_ Results_stereo.mat' is produced, which contains the information for the later removal of lens distortion in the image pairs.

9) Load the file 'Calib_Results_stereo.mat' in 'stereo_gui'.

10) Rename image pairs from date-time-position coded names to a 'stereo-gui' compatible name using the program matlab_rectify_rename_step1.exe. A file named 'filename.txt' is created in the same directory containing the original and the new file names.

11) Read the stereo-image pairs 'Load own stereo images'.

12) Remove the lens distortion with the function 'Rectifiy own stereo images'. In this step, a file called 'calib_results_stereo_rectified.mat' is created containing the final intrinsic 'fc_new' value (e.g. 4,068 pixel for the calculated distance of the lens' second nodal point and the chip plane for both cameras) and the extrinsic t_new value (e.g. 0.138 for the distance of $138 \mathrm{~mm}$ between the two optical axis of the cameras).

13) Back-rename image pairs from the 'stereo-gui' compatible MATLAB name to the original date-time-position coded name by using the program matlab_rectify_ rename_step2.exe.

Step 14: Stereoscopic

14) Use the Java-program 'StereoMarker.jar' to measure distances in image pairs. point that is offset from the centre of the pixel array of the sensor. Therefore, even with a perfect alignment of the 'object in infinity' in both cameras, a systematic error still remains in the system, which has to be estimated by a proper calibration procedure (as described in the present paper).

Once the correct position of both cameras was determined, both cameras were fixed to the baseplate by the tripod screws and, if necessary, were fixed by gluing or by screwing additional stabilising structures (e.g. small PVC or metal sticks). This procedure is recommended for the $x$-dimension. In the authors' experience, the deviation in optical axis parallelism in the $y$-dimension was always minor (less than 10 pixels) in both systems and therefore was not compensated for.

For the stereoscopic image assessment of either a moving object or a moving camera system (e.g. when operated by a scientific diver), it is crucial that both cameras are triggered simultaneously. Depending on the cameras used, simultaneous triggering can be achieved either electronically (Fischer et al., 2007) or mechanically (Klimley and Brown, 1983). In the low-cost system, the present study used a mechanical system composed of a horizontal lever above the cameras (Fig 1) with two micrometre screws above each housing trigger.

Adjustment of the screws leads to an earlier or later release of the camera. The process of synchronising the point of release of the two cameras can be achieved using a single flashlight. Using an exposure time of about $1 / 200$ s for both cameras, the trigger of the camera without flashlight should then be attuned with the micrometre screw so that the flash illuminates the image of this camera. Because a single flash has a duration of less than $5 \mathrm{~ms}(1 / 200 \mathrm{~s})$, this ensures that the two cameras are properly synchronised.

Synchronisation in the SLR system was achieved using the remote-control release connector, which is available in almost every SLR camera. According to tests and the relevant URLs on the synchronous remote-control release of multiple cameras, this allowed a synchronisation time of the cameras 
$<5 \mathrm{~ms}$. In the present study's setup, however, this was only true when no flashlight was connected. When a single flash was connected to one of the cameras, a resynchronisation of the release of up to $60 \mathrm{~ms}$ was observed. Therefore, an electronic timeshift device (Fig 2) was developed that allowed the release of one camera to be shifted in steps of $1 \mathrm{~ms}$, compared to the second camera, to achieve full synchronisation. A circuit diagram and parts list for such a time-shift device can be downloaded from the Pangea website (http://dx.doi.org/10.1594/ PANGAEA.782365).

After assembling the stereo-camera system, a strict sequential routine for image processing was applied to achieve the best results. The individual steps are listed in Table 2 and are described in detail in the following sections.

\subsection{Determining camera intrinsic and extrinsic parameters and the lens distortion matrix}

To determine the cameras' intrinsic and extrinsic parameters, a set of about 20 underwater stereo pictures of a specific calibration board with a known number of exactly defined black and white squares is required. In the present study's calibration procedure (which was a partial calibration), the calibration images were used to determine four parameters required for stereogrammetric measurements:

1) the distance between the optical axes of the two cameras in millimetres;

2) the distance from the nodal point in the cameras' lens systems to the chip planes in pixels;

3 ) the amount of misalignment of the optical axes of the two cameras to full optical parallelism in the $x$ and $y$ directions; and

4) the distortion matrix encoding the lens distortion with respect to the distortion of the pixels on the images compared to their real positions.

As stated earlier, a full calibration includes the additional elimination of the principal point offset and the decentring distortion of the lens system, which was not included in the calibration model used here. Furthermore, the use of a 2D 'checkerboard' rather than a full 3D target array is not optimal, because this type of calibration has the potential to introduce parameter correlations and thereby systematic errors.

However, the present study specifically intended to test the 2D method and its possible accuracy and precision with its camera systems, because this method is comparatively simple to apply also by non-specialists, as well as cost efficient and applicable even in rough field conditions. Even though the use of a full calibration model and a 3D target array potentially would achieve better results, the present study focused on the partial calibration, to keep the procedure as simple as possible. Based on preliminary studies with the partial calibration procedure, errors $<5 \%$ were always achieved when measuring test distances in the field. Therefore, the simple and cost-efficient method was used, because it had the potential to optimally balance out the efficiency and costs in the type of ecology field studies of interest.

All four parameters can be determined using the MATLAB toolbox 'Camera Calibration Toolbox', which is freely available, with the two routines 'calib_gui' and 'stereo_calib' (www.vision.caltech. edu/bouguetj/calib_doc). These routines are easy to use and are fully explained in the help file of the toolbox.

In the first step of calibration, the left and right images have to be copied in a single folder. Because the MATLAB routine 'calib_gui' has a very strict naming convention, the images must be renamed. All left and right images must have an identical prefix (e.g. 'left_' and 'right_'), followed by a sequential number. The number must be the same for matching stereo-images so that an identical number of left_\# and right_\# image files are present in the folder.

In the next step, all left (or right) images are read in the routine 'calib_gui' (Table 2, step 3) using the option 'load images'. For this, the identical prefix of all left images has to be provided. When all left (or right) images are successfully read in the routine, the next steps - 'extract grid corners' (Table 2, step 4), 'calibration' and 'save' - can be performed. For all steps, a detailed step-by-step procedure is given in the help file of the toolbox.

When the calibration of the left (or right) images is completed, MATLAB produces an output file called 'calib_result.mat'. This calibration file contains the intrinsic camera parameters of the left or right camera, such as the distance between the nodal point and the chip plane, as well as the lens distortion matrix. Depending on which camera (left or right) has been calibrated, this file has to be manually renamed as 'calib_result_left.mat' or 'calib_ data_right.mat' (Table 2, step 5). After this, the images of the second camera must be processed in the same way.

In the next step, a slightly modified version of the original routine 'stereo_gui', which is included in the software package from Pangea(http://dx. doi.org/10.1594/PANGAEA.782365), is used. The two calibration files (left and right) and the corresponding stereo-image pairs have to be in the same directory and read with the option 'load left and right calibration files' (Table 2, step 7). By clicking on the options 'run stereo calibration' and 'save stereo calib results', the stereo calibration is 
performed and the results are saved in the file 'Calib_Results_stereo.mat'. This file contains all the necessary information for the subsequent process of the removal of lens distortions of the image pairs.

\subsection{Processing larger numbers of stereo-image pairs}

When processing a larger number of stereo-image pairs, proper name conventions often become an important issue, especially when time series have to be analysed with many sequential images. In almost all cases, two sets of images (left and right series) potentially have the same image name (e.g. IMG0001 from the left camera and IMG0001 from the right camera). This often leads to confusion and errors, for example, when the left and right images (which are sometimes quite similar) have been accidently interchanged or wrongly matched. It is therefore highly recommended that larger sets of image pairs are renamed prior to any further processing.

The present study used the date, time and camera position (left or right) to create unique names for each image. This can be performed either manually or with a program that is able to extract this image information, for example, from the EXIF information of the images. Although the date and time (if correctly set in the cameras) are always coded in the EXIF files and can be used for later file naming, the coding of the camera position (left or right) into the EXIF file has to be performed manually before an image is shot. This is best executed using the setup menu of the camera where, for example, the name of the photographer or other image information can be set as default value for all images. In the SLR cameras used for the present study, the value $L$ (for the left camera) and $R$ (for the right camera) was set as default values for the 'copyright' in the cameras' setup menu. This information was then written in the respective EXIF tag and could be extracted subsequently for the identification of the cameras/image position.

To rename large numbers of stereoscopic image pairs in batch mode, the freeware ExifTool (www. sno.phy.queensu.ca/ phil/exiftool/) by Phil Harvey was used. This program allows the creation of unique image names using the EXIF information from the images, together with a free text. For the present study, an image name was composed using a fixed prefix 'Remos1_' followed by the date, time and image position (e.g. 'Remos1_20130631_134500_ L.jpg' for an image taken on 31 June 2013 at 13:45:00 (hh:mm:ss) with the left camera). An (annotated) example batch file for renaming images according to this name convention with the program ExifTool is available in the software package from Pangea.
After the image pairs have been named correctly, they can be corrected for lens distortion using the MATLAB routine 'stereo_gui' and the respective lens distortion matrix. In the first step, the calibration file 'Calib_Results_stereo.mat' has to be loaded using 'load stereo calib_results'. Then, the image pairs have to be loaded using the option 'load own stereo images'. This step again requires a very specific naming convention for the images, which often does not comply with practical names such as the aforementioned date-time-position coded names. The routine 'stereo_gui' requires image names with a fixed prefix (e.g. 'Remos1_'), followed by a running number '_1_' and the ID code _L or _ $\mathrm{R}$ for the left or right image, respectively (valid names are e.g. Remos1_1_L.jpg and Remos1_1_R. jpg, Remos1_2_L.jpg, etc.). This naming convention is very strict and images will not be loaded if these naming rules are not followed.

Because it can be very time-consuming to rename larger image series by hand and the date-time information will be lost, a small stand-alone program was developed called 'matlab_rectify_rename_step1.exe'. This program is included in the software package from Pangea and allows the batch-renaming of even larger numbers of stereo-image pairs in a certain directory (Table 2, step 10). The program renames, for example, image pairs from the date-timeposition coded name with the MATLAB-compatible name, but stores the original date-time-position coded names in a file called 'filename.txt' in the same directory. Furthermore, the program checks whether a left $(\mathrm{L})$ and right $(\mathrm{R})$ image is available for each pair and gives a prompt when this is not the case for a certain image pair.

When all images have been successfully renamed and loaded in 'stereo_gui', the lens correction process can be performed with the option 'rectify own stereo images' (Table 2, step 12). This step then creates: (1) rectified image pairs in bitmap format (.bmp), which can be converted to jpeg-format with the option 'convert rectified images to jpg'; and (2) the file 'calib_results_stereo_rectified.mat'. This file contains the final value for the distance between the optical axes 't_new' (e.g. t_new = -0.138 means a distance of $138 \mathrm{~mm}$ ) and the new jointly calculated distance between the image sensor and the second nodal point of the lens 'fc_new' (e.g., fc_new $=4,068$; given in pixelS) for both cameras in the stereo system. These two values are required in the final step - the measurement of an object's length or the distance between objects.

Because it might be important for a time series to ultimately retrieve the date- and time-coded image names for further analysis and not to work with the MATLAB image names, a second small 
program was developed for the present study called 'matlab_rectify_rename_step2.exe' (included in the software package from Pangea), to rename the MATLAB image names with their original date-time coded names after the lens correction is performed (Table 2, step 13).

\subsection{Measuring objects on stereo-images}

Measuring an object's size on a pair of stereo-images can basically be performed by any program that is able to extract $x y$ pixel coordinates from images. Using the 3D algorithms from Klimley and Brown (1983) adapted for computer analysis, the length and position of any object in the 3D realm can be calculated using standard geometry. Based on this algorithm, a Java program called 'StereoMarker.jar' (included in the software package from Pangea) was created for easy measurement of the lengths of objects in stereo-image pairs. The program works with the aforementioned standard naming convention, 'Prefix_yyyymmdd_hhmmss_L.jpg', and automatically loads the correct image of the right-hand camera, when it is available, into the same folder.

Prior to measuring objects, the parameters $\mathrm{fc}_{-}$ new and t_new (from the file 'calib_results_stereo_ rectified.mat') have to be provided in the program mask. After clicking an object's front on the left image, the epipolar lines (Sturm et al., 2010) of this position are imaged in the right-hand image, so that the position can easily be identified in the corresponding stereo-image, even when there are other similar objects close to the target object (e.g. in a fish swarm). The image analysis program provides all the raw data of an object ( $x y$ coordinates) as well as its length (and height if measured), plus additional free comments in a universal csv-file format.

\subsection{Experimental settings}

To assess the effects of the different camera types and settings on the accuracy and precision of length measurements in the $x, y$ and $z$ dimensions, the present study tested the two camera systems with different setups in the laboratory and in situ. In laboratory experiments, a $290 \times 210 \mathrm{~mm}$ chequered board with $29 \times 29 \mathrm{~mm}$ black and white squares was used as a target. The board was laminated and glued to a $20 \mathrm{~mm}$ PVC plate, which could be positioned at discrete distances from the cameras in the underwater test facility.

With this setup, both systems were tested with respect to the accuracy and precision of measuring discrete lengths on the image. To approximately cover the length-ranges of fish in the sampling area, the present study measured one, three and seven lengths of the edges of the squares on the chequered board in the horizontal $\left(0^{\circ}\right)$ and vertical $\left(90^{\circ}\right)$ directions, representing object lengths of $29 \mathrm{~mm}$, $87 \mathrm{~mm}$ and $203 \mathrm{~mm}$. Furthermore, diagonal length measurements were tested using the slants of the one, three and seven squares $\left(45^{\circ}\right)$, which represent object lengths of $41 \mathrm{~mm}, 123 \mathrm{~mm}$ and $288 \mathrm{~mm}$. Measurements of one, three and seven fields of the chequered board horizontally, vertically or diagonally are subsequently referred to as $1 \mathrm{~F}, 3 \mathrm{~F}$ and $7 \mathrm{~F}$.

To test the effects of an object that is not parallel to the chip plane but has a certain $z$-dimension (a fish swimming towards the camera), the same measurements were performed again, but with the two tilt-angles $30^{\circ}$ and $60^{\circ}$ of the chequered board towards the sensor chip plane. This entire setup resulted in 27 different scenarios: three main scenarios $\left(0^{\circ}, 45^{\circ}\right.$ and $90^{\circ}$ relative to the horizon) with three different measurement lengths $(1 \mathrm{~F}, 3 \mathrm{~F}$ and $7 \mathrm{~F}$ in the graphs). These scenarios were each implemented at a tilt-angle $0^{\circ}, 30^{\circ}$ and $60^{\circ}$.

In the low-cost system, the effects of a WAC and the multiple mounting and dismounting of the cameras from the rack were also tested. These effects were not tested in the SLR system, because a WAC was not used in this system and the system was never dismounted for retrieving the images. For all setups, the present study performed identical measurements with and without rectifying the images, to assess the effects of lens distortion on accuracy and precision in both systems.

\subsubsection{First setup (standard setup)}

In the first setup, five pairs of stereo-images were made with the chequered board: (a) mounted parallel to the image sensor; $(b)$ at a tilt angle of $30^{\circ}$ to the image sensor; and $(c)$ at a tilt angle of $60^{\circ}$ to the image sensor.

Using these stereo pairs, the study measured the lengths of one, three and seven squares $(2.9 \mathrm{~cm}$, $8.7 \mathrm{~cm}$ and $20.3 \mathrm{~cm}$, respectively) at $0^{\circ}$ (horizontal), $45^{\circ}$ and $90^{\circ}$ orientations (relative to the horizon; see Fig 3 for an example of the three squares measured with the chequered board at $60^{\circ}$ relative to the image sensor) at each angle $\left(0^{\circ}, 30^{\circ}\right.$ and $\left.60^{\circ}\right)$ of the chequered board. Each single measurement was performed in triplicate to estimate the accuracy of the stereo system and determine the precision of the measurements.

\subsubsection{Second setup}

For the second setup, the image pairs from the first setup were used after the removal of lens distortion and the identical measurements were performed.

\subsubsection{Third setup (WAC setup)}

The WAC setup was performed only with the lowcost system because the SLR system already had an 


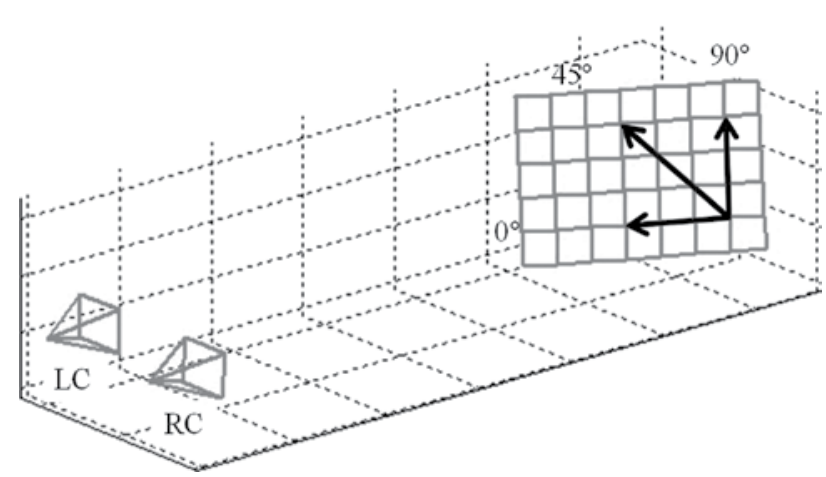

Fig 3: A schematic diagram of the various orientations of the measured objects relative to the stereo-camera ( $L C=$ left camera; $\mathrm{RC}=$ right camera). The chequered board in this example is turned $30^{\circ}$ relative to the image sensor plane of the two cameras

adjustable focal length down to $18 \mathrm{~mm}$. In contrast, low-cost cameras often only come with a standard focal length that is not practicable underwater, especially under poor visibility conditions. In many non-SLR camera systems for underwater application, additional WACs for underwater imaging are used to achieve a closer distance to the object without overly narrowing the area of view.

In this setup, the basic experiment (first setup) was repeated, but using an additional WAC (Epoque DLC-20, conversion factor 0.56, adapter ring 52-55) attached to the low-cost system.

\subsubsection{Fourth setup (object-camera distance)}

The fourth experimental setup was again performed with both camera systems and the influence of an object's distance from the camera on the accuracy and precision of the system was tested. It repeated the second setup with a distance of $45 \mathrm{~cm}$ between the chequered board and the camera systems, with a distance of $30 \mathrm{~cm}$ and $55 \mathrm{~cm}$ between the object and the cameras. Based of the results of experiments from the first and second setup, measurements at orientations of $0^{\circ}$ and $60^{\circ}$ relative to the sensor plane were performed to focus on the extreme values.

\subsubsection{Fifth setup (system mounting/handling effects)}

Similar to the third setup, the fifth setup was performed only with the low-cost system. To evaluate the stability of the camera geometry, this experiment tested the error caused by repeated mounting and dismounting of the cameras within the supplemental underwater housing. Therefore, a set of five stereo-image pairs was taken and the cameras were removed from the housing to download the pictures. Subsequently, the cameras were remounted in the housings and another five pictures were taken. This procedure was repeated three times, and changes in measurements among the individual trials were analysed. Because the SLR cameras were mounted in a single housing and picture downloading was performed via a TCP/IP connection, no such test was necessary.

\subsection{In situ application}

After the laboratory phase, both systems were tested for handling and applicability of taking stereomeasurements in situ. For this test, the low-cost system was operated by a SCUBA diver who exposed fish bait on the ground off Helgoland (southern North Sea) at a water depth of $5 \mathrm{~m}$ and took a series of stereo-images as soon as a fish came close to the bait and tried to feed on it. Three replicate experiments were performed on different days, measured a total of 1,371 fish, and analysed these data for length-frequency composition.

Both cameras were set to a focal length of $5 \mathrm{~mm}$ (standard setup) and were operated in auto mode with respect to focus, aperture and shutter speed (these cameras were not intended to be manually adjusted). Furthermore, the internal flash was turned off. These settings were adequate to completely illuminate the chequered board at the target distances.

The SLR system was integrated into a remotecontrolled underwater observatory located at Helgoland at a water depth of $5 \mathrm{~m}$ in a sandy and rocky area. The system was adjusted to take stereo-images every $30 \mathrm{~min}$ for $24 \mathrm{hr}$ from 7 May to 10 June 2011 to assess the diurnal dynamic in fish abundance and species composition in the test area off Helgoland. Images were automatically transferred to the laboratory via the Internet and were evaluated on a daily basis in two steps. First, each image was scanned manually for 5 s for the presence of any organisms, and then the organisms were identified at the species level and measured.

In this setup, both cameras were operated in manual mode with respect to focus $(18 \mathrm{~mm})$, aperture (11) and shutter speed (1/250). The internal flash was turned off and an external system flash Canon EX550 was used.

\subsubsection{Nomenclature}

In agreement with Joint Committee for Guides in Metrology (JCGM, 2008) and Fischer et al. (2007), the terms accuracy and precision in the present study are defined as follows: The accuracy (error) is the percentage deviation between the measured size of an object and its real size; zero is the lowest and best possible value. The higher the absolute value (positive or negative), the worse the accuracy is. The precision is the spread of the measured values around the measured object's size and is indicated by standard error bars. 


\section{Results}

\subsection{Basic setup}

The results of the measurements performed with the low-cost system without removing the lens distortion revealed a maximum accuracy error of $10.2 \%$ in the $1 \mathrm{~F} \_0^{\circ}$ scenario (Fig $4 \mathrm{a}$ ). The accuracy of the error decreased both when objects became larger and when they turned from horizontal to vertical, and resulted in a mean accuracy error of $-0.6 \%$ averaged over all scenarios. The precision of the measurements was good, with a maximum spread of $2.1 \%$ in the $1 F_{-} 0^{\circ}$ scenario and a mean precision of $0.5 \%$ over all scenarios.

In the identical setup, the SLR system showed significantly better accuracy, with a maximum error of only $-5.4 \%$ in the $3 \mathrm{~F}_{-} 0^{\circ}$ scenario (Fig $4 \mathrm{~d}$ ) and a mean accuracy error of $-0.1 \%$ averaged over all scenarios. The precision of the measurements with the SLR system was very similar to that of the compact system, with a maximum value of $1.9 \%$ in the $1 \mathrm{~F} \_0^{\circ}$ scenario and a mean precision value of $1.1 \%$ averaged over all scenarios.
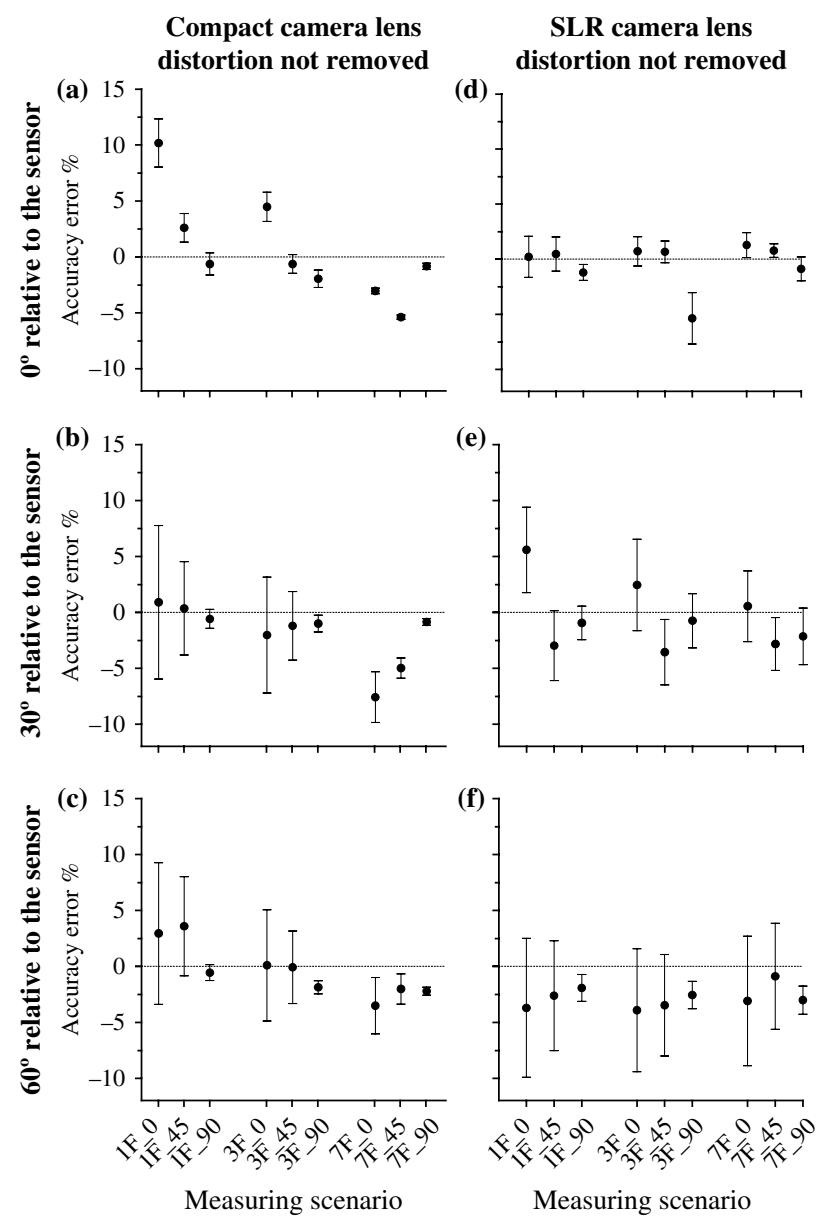

Fig 4: The values for accuracy (percentage deviation between the measured size of an object and its real size) and precision (indicated through the standard error bars derived from five replicates) made on image pairs without removing the lens distortion
When the object's orientation relative to the sensor-chip plane changed from parallel (Fig 4a,d; $0^{\circ}$ ) to $30^{\circ}$ or $60^{\circ}$, the accuracy remained more or less constant in the compact camera system (Fig 4b,c) but decreased slightly in the SLR system (Fig 4e,f). However, in both systems, the precision deteriorated (compact cameras Fig 4b,c; SLR cameras Fig 4e,f).

\subsection{The effects of removal of lens distortions}

The removal of the lens distortions resulted in a significant decrease in maximum accuracy errors in the compact system to only $-2.7 \%$ in the $60^{\circ}$ and $7 \mathrm{~F}_{-} 0^{\circ}$ scenario (Fig 5c) and a mean value of $-1.5 \%$ integrated over all scenarios.

The same improvement was observed for the SLR system, where a maximum accuracy error of $-5.2 \%$ was observed after the removal of lens distortions in the $60^{\circ}$ and $3 \mathrm{~F} \_45^{\circ}$ scenarios (Fig 5f), with a mean error of $-1.9 \%$ over all scenarios. Furthermore, the removal of the lens distortion mitigated the effect causing the measured lengths of
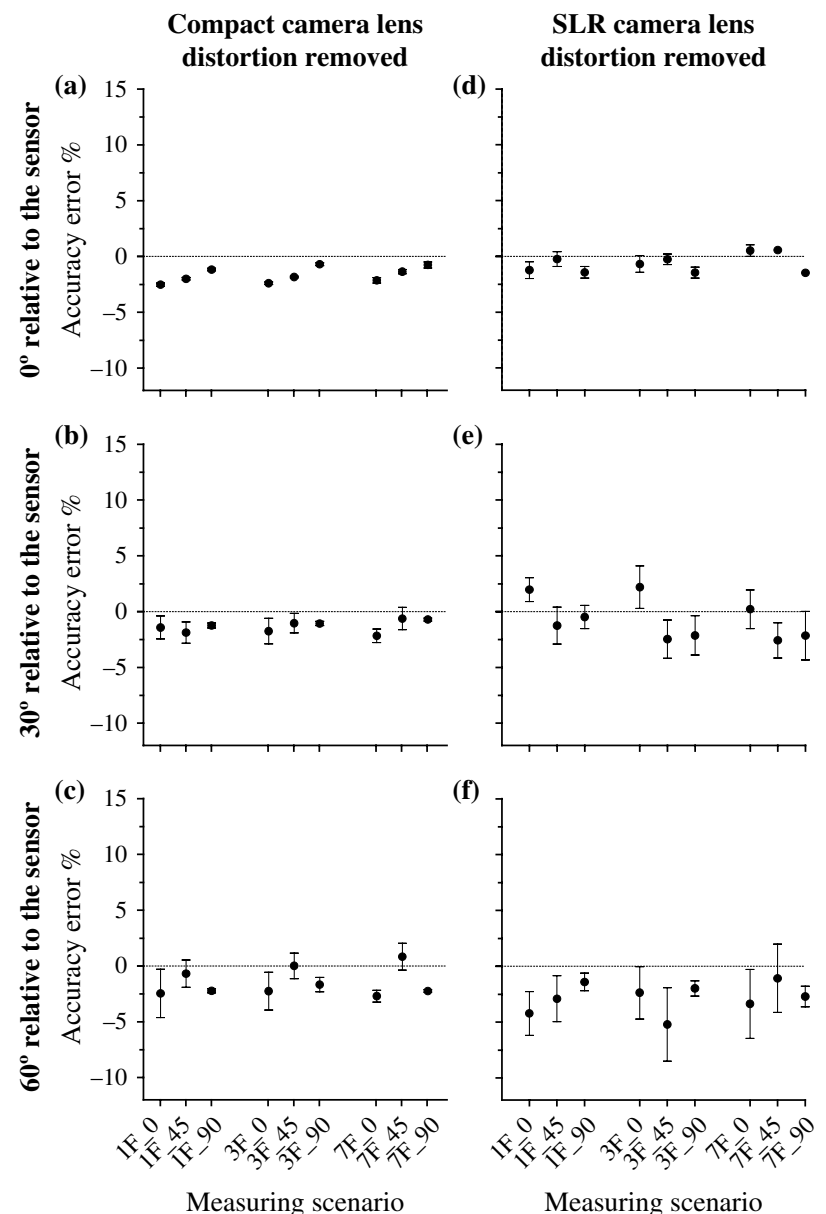

Fig 5: The values for accuracy (percentage deviation between the measured size of an object and its real size) and precision (indicated through the standard error bars derived from five replicates) made with image pairs after removing the lens distortion 
small objects (1F scenarios) to be too long and those of long objects (7F scenarios) to be too short.

Besides these accuracy improvements, a significant change was also observed in precision. By removing the lens distortion, the mean precision in the compact system integrated over all setups (Fig 5 a-c) improved to $0.1 \%$ in the compact system and to $1.6 \%$ in the SLR system (Fig $5 \mathrm{~d}-\mathrm{f}$ ). This improvement is most remarkable because in theory, measuring precision should be independent from lens distortion.

\subsection{The effects of using a wide-angle converter}

Comparing the measurements on lens distortioncorrected images taken with and without a WAC (compare Fig 5a,c with Fig 6c,d; the setup was performed only for the compact system), a slight improvement in the mean accuracy, from $-1.5 \%$ (without WAC) to $-0.5 \%$ (with WAC; Fig 6c) over all measurements was observed. However, the individual scenarios showed a larger variability when using a WAC with maximum accuracy values up to $5.2 \%$ in the $0^{\circ} \_1 \mathrm{~F}$ scenarios (Fig 6c).

In contrast, the overall precision remained more or less constant at $0.2 \%$ without a WAC and $0.3 \%$ with a WAC. Significant differences in accuracy and precision of results with and without WAC, however, were observed when the image pairs were not corrected. Both accuracy and precision deteriorated
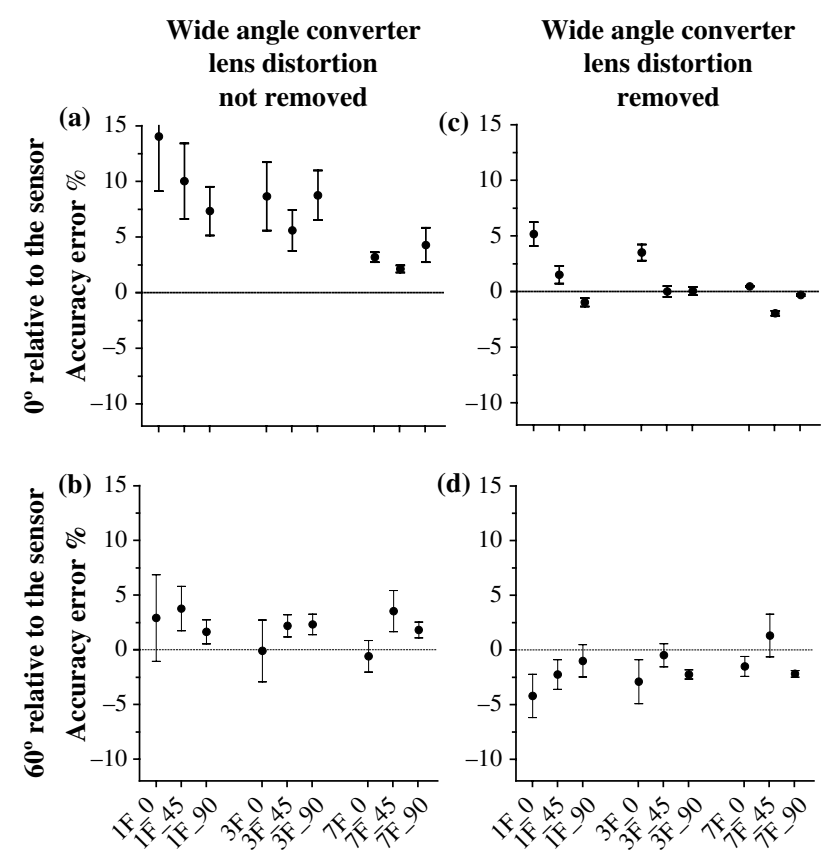

Measuring scenario

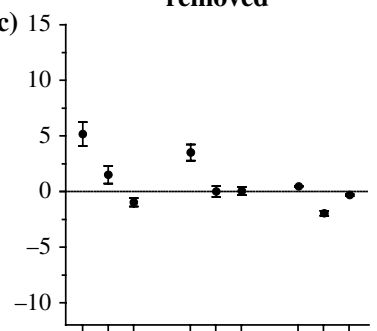

Fig 6: The effects of a wide-angle converter (WAC) on accuracy and precision compared with images with a WAC, but with removing the lens distortion significantly, with maximum accuracy errors up to $14.8 \%$ and lower precision values up to $5 \%$.

\subsection{The effects of varying distances}

The basic setup for close-up measurements $(45 \mathrm{~cm}$ between the object and camera) was derived from the comparatively short distances required for any photography assessment in frequently turbid waters in boreal coastal water bodies, especially for the assessment of small objects such as macrocrustaceans, jellyfish or juvenile fish. To obtain at least an idea of the effect of the distance between the camera system and the object on the results, images were captured in identical setups but at distances of $30 \mathrm{~cm}$ and $55 \mathrm{~cm}$. For this, the scenario from the second setup (without a WAC and with removed lens distortions) was selected, which showed the largest mean errors in accuracy and precision with the compact system, and repeated these measurements with $30 \mathrm{~cm}$ and $55 \mathrm{~cm}$ distance.

Fig 7 shows the summarised results of these measurements for the compact system (Fig 7a) and the SLR system (Fig 7b) as the mean accuracy and precision integrated over the nine measuring scenarios with one, three or seven quadrant-lengths in each orientation $\left(0^{\circ}, 45^{\circ}\right.$ and $90^{\circ}$ relative to the horizon). The results show that in the compact system, the accuracy deteriorated slightly with increasing distance from $-1.6 \%(30 \mathrm{~cm})$ to $-1.8 \%(45 \mathrm{~cm})$ and $-2.2 \%(55 \mathrm{~cm})$, whereas the precision remained almost stable at $0.6 \%$ over all distances. A similar trend for the accuracy error was observed in the SLR system with an increase from $-1.3 \%(30 \mathrm{~cm})$, to $-1.7 \%(45 \mathrm{~cm})$ and $-3.7 \%(55 \mathrm{~cm})$, and a more or less constant precision of $0.4 \%$ over all distances. (a) Compact camera

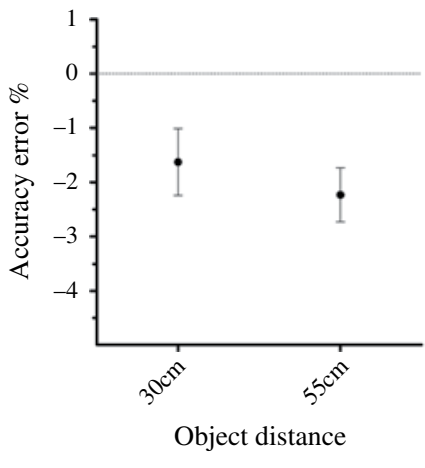

(b) SLR camera

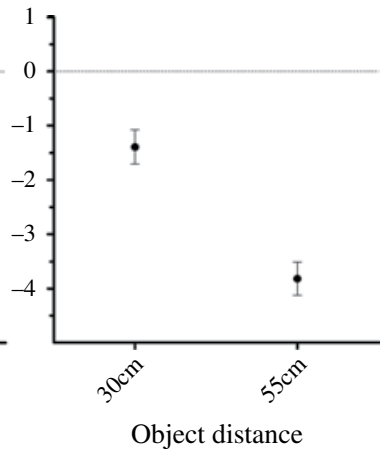

Fig 7: The effect of the distance between the camera and the object on the accuracy error and precision (a) for the low-cost system and (b) for the SLR system. The accuracy and precision are expressed as the means of values from nine measuring scenarios: measurements over one, three and seven fields at $0^{\circ}, 45^{\circ}$ and $90^{\circ}$ relative to the horizon and at $60^{\circ}$ relative to the sensor chip from two different distances 


\subsection{The effects of removing the cameras from} the underwater housings and remounting them In this setup, the present study estimated the handling-based error caused by removing compact cameras from their waterproof housings and reassembling them for data download and battery recharge. Fig 8 shows that this procedure had almost no effect on the accuracy and precision values in this setup. After the first remounting of the camera, an accuracy error of $-1.5 \%$ was detected. After the next removal-remounting step, a slight change to $-1.3 \%$ was observed. After the last removalremounting step, the accuracy returned to the initial value of $-1.5 \%$.

Similarly, the precision did not change significantly: $0.4 \%$ at the beginning, $0.5 \%$ after the first removal-remounting step and $\pm 0.4 \%$ after the last removal-remounting step. Therefore, the stereocamera system demonstrated robustness towards misalignments during this type of installation work.

\subsection{In situ application}

In the final setup, the present study tested the applicability of the two systems in situ. Fig 9 shows a typical application of the hand-held system. With a total of 25 scientific dives, an assessment of the mean (standard) length distribution of the goldsinny (Ctenolabrus rupestris (L.)) off Helgoland in October/November 2011 was performed. The images were taken by a research SCUBA diver around Helgoland Island at a water depth of between $5 \mathrm{~m}$ and $12 \mathrm{~m}$.

During these dives, a total of 2,304 stereo-image pairs were made. Detailed analysis of the image

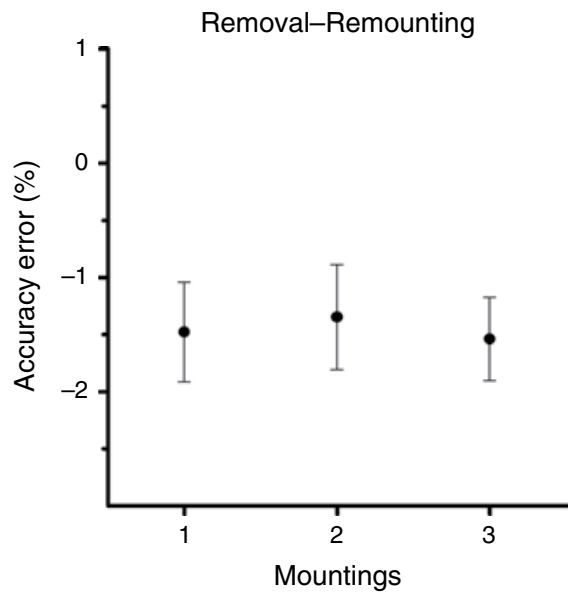

Fig 8: The effects of the repeated removal and remounting of the compact cameras from the underwater housings. The accuracy and precision are expressed as the means from nine measuring scenarios: lengths of one, three and seven fields at $0^{\circ}, 45^{\circ}$ and $90^{\circ}$ relative to the horizon and at $60^{\circ}$ relative to the sensor chip

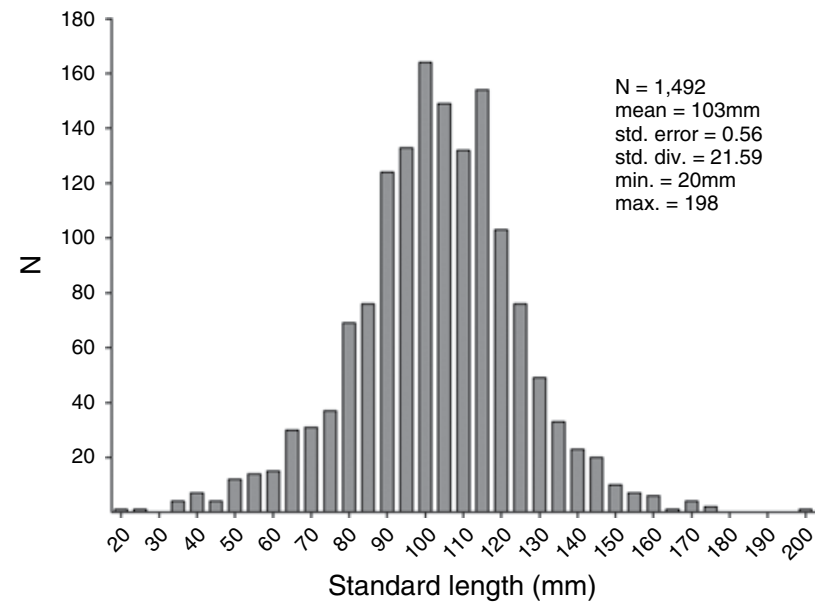

Fig 9: Non-destructive assessment of the length frequency distribution of the goldsinny population (Ctenolabrus rupestris) in the nature reserve Helgoland Felssockel. The assessment was done by scientific diving during 25 dives with the low-cost handheld stereo-camera system

pairs revealed a total of 3,065 fish that were mapped onto the image pairs, out of which 1,371 individuals could be sized. In 228 image pairs, fish could not be sized because they were not completely mapped onto both images. In another 254 image pairs, fish were mapped in a way that did not allow for precise sizing, for example, when the fish was oriented directly towards the camera so that its rear part was not visible.

A typical application of the remote-controlled SLR system with detailed abundance, species composition and length measurements of fish at Helgoland South Harbour over the diurnal cycle is shown in Fig 10. The SLR system was installed at a water depth of $5 \mathrm{~m}$ from 7 May to 10 June 2011 and took stereo-images every $30 \mathrm{~min}$ for $24 \mathrm{hr}$. Image pairs were continuously transferred via a fibre-optic underwater cable to the laboratory. The SLR system itself was completely remote controlled during this time period.

Analysis of these SLR image pairs revealed 698 sizable fish in 1,632 image pairs. Data analysis, furthermore, showed two different species with slightly different abundances in the area: the goldsinny (C. rupestris) and gobies of the genus Pomatoschistus. Both species showed a clear diurnal cycle, being active only from $06: 00 \mathrm{hr}$ to $22: 00 \mathrm{hr}$, whereas no fish were observed during the night, indicating a distinct diurnal cycle.

\section{Discussion}

The advantages of stereo-imaging in quantitative in situ assessments have been described in various studies using either analogue (Van Rooij and Videler, 1996; Evans and Norris, 1997; Bräger et al., 1999; 


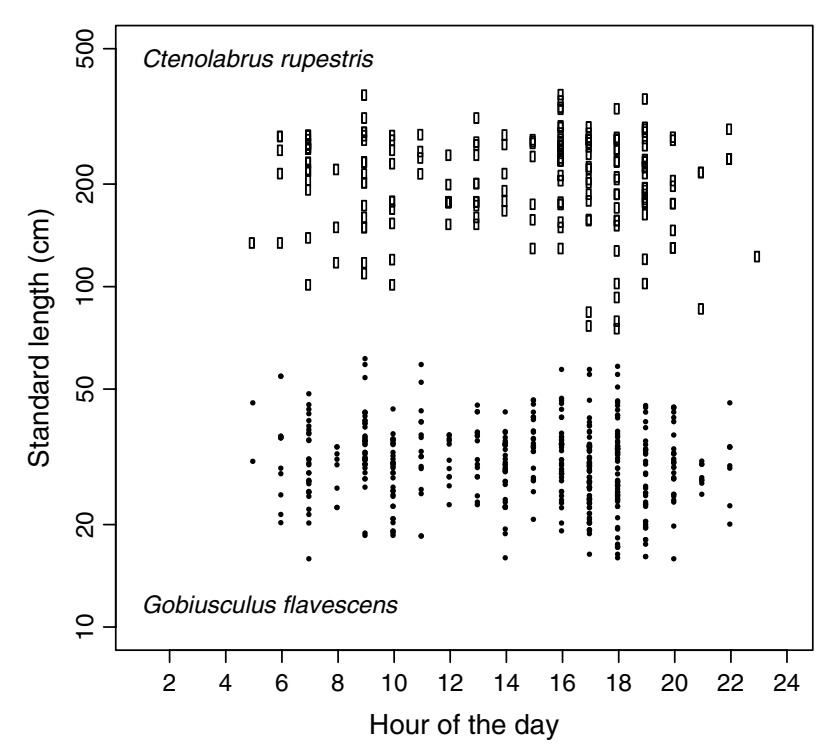

Fig 10: Non-destructive assessment of the diel occurrence of fish species ( $\square=$ Ctenolabrus rupestris, $(\bullet=$ Gobiusculus flavescens), species abundance (number of symbols per hour) and standard length distribution (centimetre) at a hard bottom substratum in the nature reserve Helgoland Felssockel. The assessment was done with the SLR system over a period of about one month in May to June 2011 using an image frequency of 30min over $24 \mathrm{hr}$ and was completely remote controlled

Svane et al., 2009) or digital (Abdo et al., 2006; Fischer et al., 2007; Williams et al., 2010) stereophotography systems. The rapid change from analogue to digital systems within the last decade has furthermore significantly enhanced the potential of the method. Camera systems can now be used even within the framework of long-term observatory technologies with cable connection and continuous high-speed data transfer rates.

The present study shows that both camera systems - the compact system and the SLR system - are suitably able to measure fish abundances as well as fish sizes (Figs 9 and 10) for many fish ecological studies, where most measurements in the field are performed, e.g. to the nearest centimetre. The present study has shown that such accuracies can be easily reached with in situ stereogrammetric methods even with low-cost consumer cameras.

This, and even more importantly the fact that stereogrammetry is a strictly non-invasive method (the research objects do not have to be killed for simply length measurements and abundance estimates), should be sufficient for this method to represent an alternative for data assessments when simple morphometric data (such as the length of fish or any other morphometric traits) are required.

Furthermore, stereophotography or stereo-video monitoring allows the observation or measurement of, for example, population growth repetitively over a longer period. Therefore the study of population structures and the activity pattern of a certain species reveals information about inter- and intraspecific interactions and behaviour (Aguzzi et al., 2009). The possibility of observing specific species over the complete diurnal cycle in their natural habitat is only possible with a cabled and fixed installed observatory such as the remote-controlled SLR system (Fig 10) or a video-monitoring system used by Aguzzi et al. (2009; 2011). An observation over 24hr, however, is highly recommended to complete studies on biodiversity and population assessments (Aguzzi et al., 2012; 2013).

The use of visual censuses by divers might provide an efficient opportunity to reveal information about the rhythmic activity of rocky-reef fish as shown by Azzurro et al. (2013). However, the application of SCUBA over a complete $24 \mathrm{hr}$ period requires a high manpower and acceptable environmental conditions to guarantee the safety of the diver. The hand-held system can provide an alternative to traditional census techniques, or can be used as an additional tool.

In contrast to a fixed observatory, it can be used more flexibly, e.g. to observe differences in the population structure of a specific species with respect to its habitat. Furthermore, it allows the diver to achieve a high sampling rate within a relatively short sampling time in the field. This is an advantage, especially in areas where dive time is limited owing to low temperature or strong tide currents, which is the case for large parts of the North Sea (Wehkamp and Fischer, 2013).

However, in situ stereo-optical applications are still comparatively rare, which is mainly owing to insufficient documentation of the methodology itself. Furthermore, important (optical) parameters necessary to build up a calibrated stereo-optical camera system with standard consumer cameras are almost impossible to obtain from any camera manufacturer. This leads to a situation in which available mathematical formulae for length calculations (e.g. Van Sciver, 1972; Klimley and Brown, 1983) and stereoscopic analysis software can hardly be applied without an intense and time-consuming preparation phase. Especially when using standard consumer cameras, for which the manufacturer does not provide any of the required intrinsic camera data even upon request, determining these parameters is almost impossible without a specific correct mathematical procedure.

The present paper is targeted to the aquatic scientist or technician who is planning non-invasive measurements of objects or biota underwater who does not wish to become a specialist in underwater optics, software programming, engineering 
or mathematics prior to taking measurements in the field. Both systems tested were built in the classical manner of stereogrammetry, as described in Klimley and Brown (1983) and Van Rooij and Videler (1996), with the optical axes pre-adjusted to be as parallel to each other as possible.

The results of the present study showed that this arrangement allows the determination of an object's real size, even in images that are not corrected for lens distortion, with an accuracy of about $1.5 \%$ (consumer system) and $0.9 \%$ (SLR system) for most objects and scenarios. However, the present study also showed that without correction for lens distortion, accuracy error can increase to $>15 \%$ under certain circumstances. This means that two fish with an identical length of $4 \mathrm{~cm}$ might be sized at $4 \mathrm{~cm}$ or $4.6 \mathrm{~cm}$, even when measured in the same image pair, depending on their orientation in the 3D space.

According to the results, the removal of lens distortions can be identified as an important step in improving the results in stereo-image analysis. This is best explained by comparing the same image before and after the removal of lens distortions (Fig 11). In Fig 11a of the uncorrected image, lines on the calibration board at the outer areas of the image are much more distorted owing to optical lens aberrations and therefore are heavily bowed. An object is measured in these outer areas of the image as too long, because distances in these outer edges of the image are artificially stretched by lens aberration. Therefore, measuring objects in the outer areas of the uncorrected image may lead to lower accuracies especially when lens systems are of lower quality and generate a strong distortion of the outer image areas. Furthermore, because the same object is projected onto different areas of the left and right images of a stereo-pair, the projection of the object is located in differently distorted image areas, which is an additional source of accuracy error.

However, this effect cannot fully explain the observed differences in precision between uncorrected and corrected images of objects measured in horizontal or vertical position. Theoretically, the precision of measurements is related to the user's ability to locate an exact position on the monitor by clicking on the proper pixel with the computer mouse. Assuming that the user has a 'natural' ability to locate and click on a certain point in the image within 10 pixels (taking into account e.g. hand shakiness, the resolution of the mouse, an inappropriate display), the theoretical precision error of measurement in the compact system is $\pm 4.2 \%$ of the true value. If the same 'object' is measured while oriented at $60^{\circ}$ to the chip plane (swimming at an angle of $60^{\circ}$ towards or away from the camera), this object would be only 110 pixels in size. In this case, the theoretical precision error in measurement increases to $\pm 9.1 \%$ of the true value.

This explains the overall lower precision in measurements made from both the uncorrected and corrected images of an object that is not parallel to the chip plane. This precision is difficult to mitigate by the removal of lens distortion, but can be improved by attempting to make the object of interest as large as possible in the image.

The precision of an object parallel to the chip plane should not significantly change when removing lens distortion or when an object shifts from a horizontal to a vertical position. In the present study, however, both effects were observed in the measurement, with a significant improvement of precision after removal of lens distortion as well as when the objects shifted to an upright position.

An explanation for these changes in precision might be that the measured objects on the corrected (a)

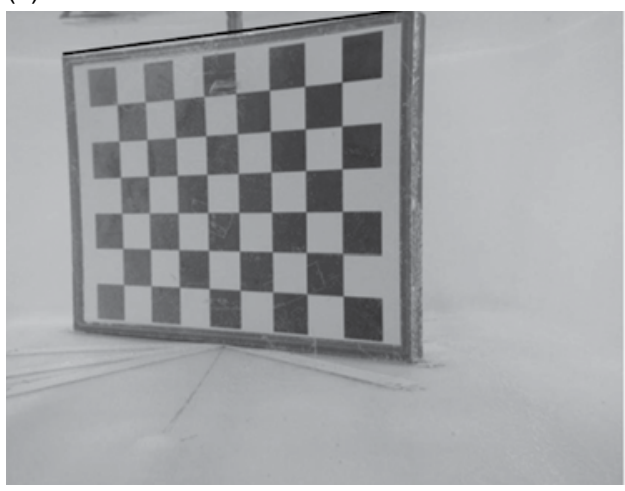

(b)

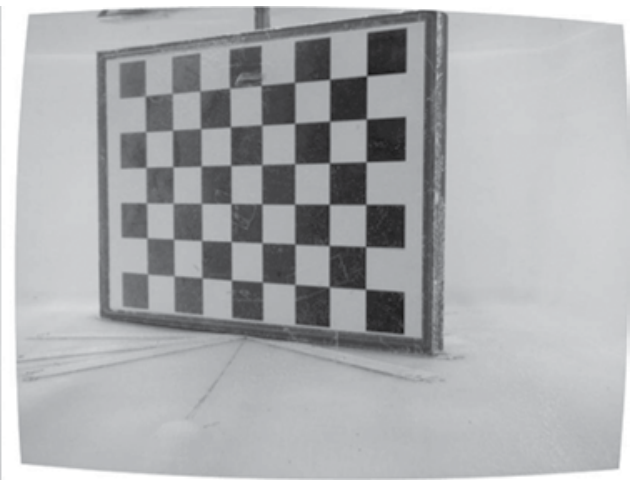

Fig 11: Image of the calibration board (a) without and (b) with removed lens distortion. The black line above the board is a true straight line. Because of the lens aberrations of the optical system of the camera, the lines at the outer areas on the test board are much more distorted compared to the central areas of the image. This leads to a significant error in size calculations 
image were often located in the centre of the image compared to the uncorrected images. The measuring setup in the present study, however, was explicitly designed as a random sampling to exclude these systematic errors, and therefore this explanation seems to not be applicable. Because there was no significant change in image quality between the different scenarios, which may be another explanation for the observed changes, further detailed studies on this topic are necessary to shed light on these yet not fully understood changes in precision.

Another shortcoming of the present study was that it could not estimate the accuracy and precision for larger distances between the camera and the objects, so that the values were basically only valid for short distances up to $60 \mathrm{~cm}$. Tests should be performed to establish the relationship between distance and accuracy for a wider range of distances.

A further point that should be studied in detail with additional data from these systems is the somewhat worse accuracy with increasing distance for the digital SLR cameras $(3.7 \%)$ compared to the compact camera system $(2.2 \%)$. The SLR system has basically the advantages of a greater image resolution, a larger format sensor, a wider base between the cameras and a longer principal distance, and therefore accuracy errors normally should be lower when compared to compact systems. The reason for the contrary results cannot be completely explained here, but might result from measuring different squares on the checker-board during accuracy and precision estimation.

The $x y$ positions of the squares measured for the different setups were selected by a fixed random sampling routine. Assuming that the calculated algorithm for removing the lens distortion by the MATLAB toolbox was not fully successful over the entire image, somewhat blurred image areas might remain even after this process. If the measured squares lie within these areas, this might lead to such effects. However, further calibration images and analyses to specifically focus on these remaining errors would be necessary. As the present paper specifically focuses on the applicability of stereogrammetry under routine conditions, with a strong focus on the range of errors occurring under such routine conditions, it has omitted a detailed analysis of such errors here.

\section{Recommendations and possible improvements}

Five out of the six setups presented in the present paper were performed under more or less controlled conditions with a non-moving object (the calibration board), which was held by a scientist diver in front of the camera system (SLR) or fixed on a bottom-plate (compact system). The 'objects' (width, diagonal and height of one, three or seven squares) were selected by a random number generator, which pulled one of the nine squares in the $x$ direction and one of the seven squares in the $y$ direction as a starting point for each measurement. When comparing the effects of the removal of lens distortions, the same random numbers and starting points were used for the uncorrected and the corrected image pairs. However, the accuracy and precision can decrease somewhat in the field, especially when measuring moving objects and therefore possibly fuzzy images.

A further source of error, which was not tested here, might be strongly reduced visibility, which occurs often in the German Bight. Unfavourable light conditions with a high refraction of light combined with fast-moving objects can lead to blurred pictures, which will be impossible to evaluate or will be evaluated with a loss of accuracy and precision. The presented data, therefore, depict the technical possibilities and limitations, but most certainly not the best results, achievable with these systems. However, in the authors' opinion, even the consumer system revealed highly accurate and precise data, which are of similar or even better quality than, for example, measurements of killed fish on a boat using standard field measurement equipment.

For the detection, for example, of fine-grained differences between groups of the same fish species and age class at different locations in a survey area, superior accuracy and precision might be necessary (Harvey et al., 2001; 2002). The results could be improved by additional hardware that fine-tunes the optical axes of the cameras, or by increased effort in calibrating the system with more calibration images (details are given in the description of the MATLAB routine 'calib_gui'; www.vision.caltech. edu/bouguetj/calib_doc).

A further important point is the camera synchronisation, especially when the scientist diver who is operating the system or the object is moving. The accuracy and precision of any stereo-image system is only as good as the synchronisation of the two cameras with respect to the shutter release. A proper synchronisation is not such a problem when a single flashlight is used, because the duration of a standard flash (and therefore the exposure time of the image) is about only $5 \mathrm{~ms}$. This means that even when the shutter of the cameras has to be open for longer (e.g. $1 / 50$ s or $1 / 20$ s) to guarantee that the flashlight fires during the time when both shutters are open, an exposure time of about $1 / 200$ s ( $1000 \mathrm{~ms}$ divided by $5 \mathrm{~ms}$ ) in terms of standard camera exposure times is realised. Because 
almost any aquatic organism can be imaged quite sharply within this exposure time, a sufficient synchronisation of the two images can be assumed when an object is projected sharply in both images.

However, when no flashlight is used, the time synchronisation has to be controlled very carefully, which can be time consuming, especially when using a manual trigger system or, even worse, a triggering system via a USB connector. In the latter case, triggering cameras via USB almost always leads to the failure of proper camera synchronisation because USB protocol does not allow precise time communication with the camera. It is therefore highly recommended to use either camera systems that can be triggered manually by some mechanical device or, much better, cameras that have a separate connector for an electronic trigger device. These devices are almost always based on the principle of creating an electrical short-circuit between two pins of that connector and allow a time synchronisation of $<5 \mathrm{~ms}$ when using identical camera systems. When working without a flash, the proper time synchronisation should be verified with the help of a single flashlight.

Concluding the described procedures and results, stereoscopic underwater assessments with either hand-held or remotely-operated camera systems can provide highly accurate measurements of objects under water. Most importantly, such measurements can be achieved without being a specialist in the field of 3D photogrammetry or applying complex mathematical algorithms. The experiments in the present paper have shown that even less skilled students or researchers can use a consumer-built stereoscopic system to make exact measurements and that stereoscopic image analysis is also feasible with existing (and mainly freely available) software. Because this method is non-destructive, organisms do not have to be killed to assess species-specific morphometric traits (such as body length), and this method should be actively promoted in aquatic ecology to foster a sustainable research approach.

\section{Acknowledgements}

The authors thank the technical engineering departments of the Alfred Wegener Institute, Helmholtz Centre for Polar and Marine Research and the Biologische Anstalt Helgoland for building several parts of the camera; Jakob Klaus-Stöhner (Company Loth engeneering) for software programming; Manoucher Doroshtnasir for MATLAB software support; several student helpers for the image analysis; Stephanie Wehkamp and two anonymous reviewers for thoroughly reading and correcting the manuscript. The work was performed within the framework of COSYNA (Coastal Observing System for the Northern and Arctic Seas).

\section{References}

Abdo DA, Seager JW, Harvey ES, Mcdonald JI, Kendrick GA and Shortis MR. (2006). Efficiently measuring complex sessile epibenthic organisms using a novel photogrammetric technique. Journal of Experimental Marine Biology and Ecology 339: 120-133.

Aguzzi J, Costa C, Fujiwara Y, Iwase R, Ramirez-Llorda E and Menesatti P. (2009). A novel morphometry-based protocol of automated video-image analysis for species recognition and activity rhythms monitoring in deep-sea fauna. Sensors 9: 8438-8455.

Aguzzi J, Costa C, Robert K, Matabos M, Antonucci F, Juniper SK and Menesatti P. (2011). Automated image analysis for the detection of benthic crustaceans and bacterial mat coverage using the VENUS undersea cabled network. Sensors 11: 10,534-10,556.

Aguzzi J, Company JB, Costa C, Matabos M, Azzurro E, Manuel A, Menesatti P, Sarda F, Canals M, Delory E, Cline D, Favali P, Juniper SK, Furushima Y, Fujiwara Y, Chiesa JJ, Marotta L, Bahamon N and Priede IG. (2012). Challenges to the assessment of benthic populations and biodiversity as a result of rhythmic behaviour: video solutions from cabled observatories. In: Gibson RN, Atkinson RJA, Gordon JDM and Hughes RN (eds.). Oceanography and Marine Biology: An Annual Review, vol. 50. Boca Raton, FL: CRC Press, 235-285.

Aguzzi J, Sbragaglia V, Santamaría G, Del Río J, Sardà F, Nogueras M and Manuel A. (2013). Daily activity rhythms in temperate coastal fishes: Insights from cableed observatory video monitoring. Marine Ecology Progress Series 486 : 223-236.

Azzurro E, Aguzzi J, Maynou F, Chiesa JJ and Savini D. (2013). Diel rhythms in shallow Mediterranean rockyreef fishes: a chronobiological approach with the help of trained volunteers. Journal of the Marine Biological Association of the United Kingdom 93: 461-470.

Bower MR, Gaines DB, Wilson KP, Wullschleger JG, Dzul MC, Quist MC and Dinsmore SJ. (2011). Accuracy and precision of visual estimates and photogrammetric measurements of the length of a small-bodied fish. North American Journal of Fisheries Management 31: 138-143.

Boyce RE. (1963). Simple scale determination on underwater stereo pairs. Deep-Sea Research 11: 89-91.

Bräger S and Chong AK. (1999). An application of close range photogrammetry in dolphin studies. The Photogrammetric Record 16: 503-517.

Bräger S, Chong A, Dawson S, Slooten E and Würsig B. (1999). A combined stereo-photogrammetry and underwater-video system to study group composition of dolphins. Helgoland Marine Research 53: 122-128.

Brandou V, Allais AG, Perrier M, Malis E, Rives P, Sarrazin J and Sarradin PM. (2007). 3D reconstruction of natural underwater scenes using the stereovision system IRIS. OCEANS 2007 - Europe, 18-21 June, Aberdeen, UK, $1-6$.

Bythell JC, Pan P and Lee J. (2001). Three-dimensional morphometric measurements of reef corals using underwater photogrammetry techniques. Coral Reefs 20: 193-199.

Chong AK and Stratford P. (2002). Underwater digital stereo-observation technique for red hydrocoral study. Photogrammetric Engineering and Remote Sensing 68: 745-751. 
Christie H and Green NW. (1982). Changes in the sublittoral hard bottom benthos after a large reduction in pulp mill waste to Iddefjord, Norway, Sweden. Netherlands Journal of Sea Research 16: 474-482.

Costa C, Loy A, Cataudella S, Davis D and Scardi M. (2006). Extracting fish size using dual underwater cameras. Aquacultural Engineering 35: 218-227.

Costa C, Scardi M, Vitalini V and Cataudella S. (2009). A dual camera system for counting and sizing Northern Bluefin Tuna (Thunnus thynnus; Linnaeus, 1758) stock, during transfer to aquaculture cages, with a semi automatic Artificial Neural Network tool. Aquaculture 291: 161-167.

Cullen JM, Shaw E and Baldwin HA. (1965). Methods for measuring the three-dimensional structure of fish schools. Animal Behaviour 13: 534-543.

Dill LM, Dunbrack RL and Major PF. (1981). A new stereophotographic technique for analyzing the three-dimensional structure of fish schools. Environmental Biology of Fishes 6: 7-13.

Doerffer R, Colijn F and van Beusekom J. (2008). Observing the coastal sea: An atlas of advanced monitoring techniques. LOICZ Reports \& Studies No. 33. Geesthacht, Germany: Helmholtz-Zentrum Geesthacht Research Centre. Available at www.hzg.de/imperia/md/content/ gkss/institut_fuer_kuestenforschung/kok/icon/ marcopoli_r_s_33.pdf, last accessed $<20$ May 2014 $>$.

Doya C, Aguzzi J, Pardo M, Matabos M, Company JB, Costa C and Milhaly S. (2014). Diel behavioral rhythms in the sablefish (Anoplopoma fimbria) and other benthic species, as recorded by deep-sea cabled observatories in Barkley canyon (NEPTUNE-Canada). Journal of Marine Systems 130: 69-78.

Emerey KO, Merrill AS and Trumbull JVA. (1965). Geology and biology of the sea floor as deduced from simultaneous photographs and samples. Limnology and Oceanography 10: 1-25.

Evans L and Norris R. (1997). Prediction of benthic macroinvertebrate composition using microhabitat characteristics derived from stereo photography. Freshwater Biology 37: 621-633.

Fischer P, Weber A, Heine G and Weber H. (2007). Habitat structure and fish: assessing the role of habitat complexity for fish using a small, semiportable, 3-D underwater observatory. Limnology and Oceanography Methods 5: 250-262.

Harvey E, Fletcher D and Shortis M. (2001). Improving the statistical power of length estimates of reef fish: a comparison of estimates determined visually by divers with estimates produced by a stereo-video system. Fishery Bulletin 99: 72-80.

Harvey E, Fletcher D and Shortis M. (2002). Estimation of reef fish length by divers and by stereo-video - a first comparison of the accuracy and precision in the field on living fish under operational conditions. Fisheries Research 57: 255-265.

Harvey E, Cappo M, Shortis M, Robson S, Buchanan J and Speare P. (2003). The accuracy and precision of underwater measurements of length and maximum body depth of southern bluefin tuna (Thunnus maccoyii) with a stereo-video camera system. Fisheries Research 63 : 315-326.

Jasiobedzki P, Se S, Bondy M and Jakola R. (2008). Underwater 3D mapping and pose estimation for ROV operations. Oceans 2008, 15-18 September, Quebec City, Quebec, 1-6.
Joint Committee for Guides in Metrology (JCGM). (2008). Evaluation of measurement data: Guide to the expression of uncertainty in measurement. Bureau International des Poids Mesures. Available at www.bipm.org/utils/common/ documents/jcgm/JCGM_100_2008_E.pdf， last accessed $<20$ May 2014>.

Klimley AP and Brown ST. (1983). Stereophotography for the field biologist - measurement of lengths and threedimensional positions of free-swimming sharks. Marine Biology 74: 175-185.

Leatherdale JD and Turner DJ. (1983). Underwater photogrammetry in the North Sea. The Photogrammetric Record 11: 151-167.

Li R, Li H, Zou W, Smith RG and Curran TA. (1996). An underwater digital photogrammetric system for fishery geomatics. International Archives of Photogrammetry and Remote Sensing 3: 524-529.

Pitcher TJ. (1975). A periscopic method for determining the three-dimensional positions of fish in schools. Journal of the Fisheries Research Board of Canada 32: 1533-1538.

Rooper CN, Hoff GR and De Robertis A. (2010). Assessing habitat utilization and rockfish (Sebastes spp.) biomass on an isolated rocky ridge using acoustics and stereo image analysis. Canadian Journal of Fisheries and Aquatic Sciences 67: 1658-1670.

Rorslett B, Green NW and Kvalvagnaes K. (1978). Stereophotography as a tool in aquatic biology. Aquatic Botany 4: 73-81.

Ruff BP, Marchant JA and Frost AR. (1995). Fish sizing and monitoring using a stereo image-analysis applied to fish farming. Aquacultural Engineering 14: 155-173.

Sawada K, Takahashi H, Abe K, Ichii T, Watanabe K and Takao Y. (2009). Target-strength, length, and tilt-angle measurements of Pacific saury (Cololabis saira) and Japanese anchovy (Engraulis japonicus) using an acousticoptical system. Ices Journal of Marine Science 66: 1212-1218.

Shortis MR, Harvey E and Abdo D. (2009). A Review of under water stereo-image measurement for marine biology and ecology applications. In: Gibson RN, Atkinson RJA and Gordon JDM (eds.). Oceanography and marine biology: An annual review. Boca Raton, FL: CRC Press, 257-292.

Shortis MR, Seager JW, Williams A, Barker BA and Sherlock M. (2007). A towed body stereo-video system for deep water benthic habitat surveys. In: Grun A and Kahmen $\mathrm{H}$ (eds.). Eighth conference on optical 3-D measurement techniques 2: 150-157.

Shortis MR, Seager JW, Williams A, Barker BA and Sherlock M. (2008). Using stereo-video for deep water benthic habitat surveys. Marine Technology Society Journal 42: 28-37.

Sturm P, Ramalingam S and Tradif J-P. (2010). Camera models and fundamental concepts used in geomatric computer vision. Foundations and Trends ${ }^{\circledR}$ in Computer Graphics and Vision 6: 1-183.

Svane I, Hammett Z and Lauer P. (2009). Impacts of trawling on benthic macro-fauna and -flora of the Spencer Gulf prawn fishing grounds. Estuarine Coastal and Shelf Science 82: 621-631.

Tillett R, Mcfarlane N and Lines J. (2000). Estimating dimensions of free-swimming fish using 3D point distribution models. Computer Vision and Image Understanding 79: 123-141.

Torisawa S, Kadota M, Komeyama K, Suzuki K and Takagi T. (2011). A digital stereo-video camera system for threedimensional monitoring of free-swimming Pacific bluefin 
tuna, Thunnus orientalis, cultured in a net cage. Aquatic Living Resources 24: 107-112.

Van Rooij JM and Videler JJ. (1996). A simple field method for stereo-photographic length measurement of free-swimming fish: merits and constraints. Journal of Experimental Marine Biology and Ecology 195: 237-249.

Van RooijJ, Bruggemann J, Videler J and Breeman A. (1995). Plastic growth of the herbivorous reef fish Sparisoma vir$i d e$. field evidence for a trade-off between growth and reproduction. Marine Ecology Progress Series 122: 93-105.

Van Sciver WJ. (1972). Scale determination of unrecognized undersea objects by stereographic photography. Marine Technology Society Journal 6: 14-16.

Waite JN, Schrader WJ, Mellish J-AE and Horning M. (2007). Three-dimensional photogrammetry as a tool for estimating morphometrics and body mass of Steller sea lions (Eumetopias jubatus). Canadian Journal of Fisheries and Aquatic Sciences 64: 296-303.

Wehkamp S and Fischer P. (2013). Impact of coastal defence structures (tetrapods) on a demersal hard-bottom fish community in the southern North Sea. Marine Environmental Research 83: 82-92.

Williams JCC. (1969). Simple Photogrammetry: plan-making from small-camera photographs taken in the air, on the ground or underwater. London: Academic Press, 211pp.

Williams K, Rooper CN and Towler R. (2010). Use of stereo camera systems for assessment of rockfish abundance in untrawlable areas and for recording pollock behavior during midwater trawls. Fishery Bulletin 108: 352-362. 